\title{
Heparanase is involved in the proliferation and invasion of nasopharyngeal carcinoma cells
}

\author{
JINZHANG CHEN $^{1 *}$, DAYONG ZHENG ${ }^{1 *}$, JIE SHEN $^{3 *}$, JIAN RUAN $^{1}$, AIMIN LI $^{1}$, WENMIN LI ${ }^{1}$, \\ GUOZHU XIE ${ }^{2}$, XIAOJUN LUO ${ }^{1}$, PENG ZHAO $^{4}$ and HANG ZHENG ${ }^{1}$ \\ Departments of ${ }^{1}$ Oncology, ${ }^{2}$ Radiation Oncology, Nanfang Hospital, Southern Medical University, \\ Guangzhou, Guangdong 510515; ${ }^{3}$ Department of Cell Biology, Southern Medical University, \\ Guangzhou, Guangdong 510515; ${ }^{4}$ Department of Medical Oncology, The First Affiliated Hospital, \\ School of Medicine, Zhejiang University, Hangzhou, Zhejiang 310003, P.R. China
}

Received November 26, 2012; Accepted December 28, 2012

DOI: $10.3892 /$ or.2013.2325

\begin{abstract}
Heparanase (HPSE), an endo- $\beta$-D-glucuronidase, is overexpressed in nasopharyngeal carcinoma (NPC). The purpose of our study was to investigate the possible role of HPSE in the development of NPC. RNA interference (RNAi) using an HPSE small hairpin RNA (HPSE shRNA) was used to identify the effects of HPSE on the regulation of the malignant behaviors of NPC. CNE-2, a highly metastatic human NPC cell line in which HPSE mRNA and protein levels were detected to be the highest in three NPC cell lines involved in the research, was selected as a cell model in vitro and in vivo. The results showed that downregulation of HPSE significantly inhibited the proliferative and invasive abilities of CNE-2 cells partially through MAPK signaling. Compared with the parental NPC cells, HPSE-silenced cells exhibited attenuated capacity for developing tumors in nude mice, while the growth of tumor xenografts derived from these cells was dramatically suppressed. In conclusion, our results suggest that HPSE contributes to the proliferation and metastasis of NPC, and HPSE may be a potent molecular target for NPC treatment.
\end{abstract}

Correspondence to: Professor Hang Zheng, Department of Oncology, Nanfang Hospital, Southern Medical University, Guangzhou, Guangdong 510515, P.R. China

E-mail: zhaojia233@163.com

Dr Peng Zhao, Department of Medical Oncology, The First Affiliated Hospital, School of Medicine, Zhejiang University, Hangzhou, Zhejiang 310003, P.R. China

E-mail: zhaop6@126.com

*Contributed equally

Key words: nasopharyngeal carcinoma, heparanase, invasion, proliferation

\section{Introduction}

Nasopharyngeal carcinoma (NPC) is a distinct malignancy that differs in epidemiology from other types of cancers, most frequently occurring in South-Eastern Asia (1). Despite advances in the therapy of NPC, no significant improvements have been observed in terms of overall survival and relapsefree survival due to local or regional failure and recurrence (2). Thus, the mechanism underlying the oncogenesis and development of NPC remains to be elucidated.

Heparanase (HPSE), an endo- $\beta$-D-glucuronidase, is able to cleave heparan sulfate proteoglycans (HPSG) within the extracellular matrix (ECM), basement membrane (BM) or on the cellular surface, influencing cell growth and invasion of many malignancies by regulating the shedding of HPSG (3). Elevated expression of HPSE was observed in head and neck tumors, pancreatic tumors, hepatocellular carcinoma, and esophageal carcinomas (4). Inhibition of HPSE in lymphoma, glioma, breast carcinoma and melanoma cells displayed significantly attenuated adhesion and invasion capacities (5). In addition, gene silencing of HPSE efficiently suppresses the proliferation, invasion, metastasis and angiogenesis of gastric cancer cells (6). Silencing of HSPE also resulted in the remarked inhibition of the growth of prostate tumors (7).

A previous study demonstrated that HPSE overexpression in nasopharyngeal carcinoma tissues is inversely correlated with patient survival (8); however, it remains largely unknown whether inhibition of HPSE expression affects the biological function of nasopharyngeal carcinoma cells. This study analyzed the effects of an shRNA targeting HPSE on cultured NPC cells. Our results indicated that downregulation of HPSE expression abolished the growth and invasion of NPC cells.

\section{Materials and methods}

Cell lines and culture conditions. Nasopharyngeal carcinoma cells C666-1, 6-10B and CNE-2 were kindly provided by the Oncology Laboratory of Nanfang Hospital (Guangzhou, China). All cells were maintained in DMEM with $10 \%$ FBS (both from Invitrogen/Gibco). 
$R N A$ isolation and $q R T-P C R$. Total cellular RNA was isolated from 3 NPC cell lines using TRIzol reagent (Invitrogen) according to the manufacturer's instructions and was quantified using a UV spectrophotometer. RNA $(2 \mu \mathrm{g})$ was reverse transcribed using the Access Reverse Transcription system (Promega, Madison, WI, USA) according to the standard protocol. In brief, reaction mixtures (total volume, $20 \mu \mathrm{l}$ ) containing $500 \mathrm{ng}$ cDNA were amplified to a final concentration of $250 \mathrm{nM}$ using $10 \mu \mathrm{l}$ of the $2 \mathrm{X}$ Brilliant SYBR-Green QPCR Master Mix kit (Stratagene). The primers were: HPSE forward, 5'-GAATGGACGGACTGCTAC-3'; HPSE reverse, 5'-CCAAAGAATACTTGCCTCA-3' (GenBank: NM_006665.5) (6); $\beta$-actin forward, 5'-TAAGAAGCTGCTG TGCTACG-3'; $\beta$-actin reverse, 5'-GACTCGTCATACTCC TGCTT-3' (GenBank: NM_001101). Morever, thermal cycling conditions were as follows: $94^{\circ} \mathrm{C}$ for 5 min and 45 cycles at $94^{\circ} \mathrm{C}$ for $30 \mathrm{sec}$, followed by $60^{\circ} \mathrm{C}$ for $30 \mathrm{sec}$ and $72^{\circ} \mathrm{C}$ for $45 \mathrm{sec}$. Experiments were performed in triplicate in the same reaction. Target genes and the $\beta$-actin gene were amplified in the same reaction. The results of the relative quantitation were analyzed by comparison of $2^{-\Delta \Delta \mathrm{Ct}}$.

Vector construction and transfection. Three pairs of shRNAs were designed (GenePharma) according to the HPSE sequence in the GenBank (NM_006665.5) to verify the specific effect of HPSE on the biological function of NPC, as shown in Table I. Scrambled shRNA (pGPU6/GFP/Neo-shNC) was set as the negative control. Each vector contained the neomycinresistance gene to provide neomycin resistance in mammalian cells. Efficiency of interference was evaluated by qRT-PCR and by western blot analysis. The chosen constructed pGPU6/ GFP/Neo-HPSE-shRNAs were introduced into CNE-2 cells using Lipofectamine reagent (Invitrogen). Stable cell lines selected with media containing $400 \mu \mathrm{g} / \mathrm{ml} \mathrm{G} 418$, were named CNE-2/shHPSE and CNE-2/NC. HPSE, ERK, p-RAF, p-ERK and $\mathrm{p}$-MEK expression levels for each group were detected using western blot analysis.

Western blot analysis. Cell samples were lysed in a lysis buffer (Beyotime, Jiangsu, China) after collection from a $100-\mathrm{mm}$ dish and disruption, respectively. Proteins $(20 \mu \mathrm{g})$ were resolved on $10 \%$ SDS-PAGE and transferred to PVDF membranes. Western blot analysis was performed using antibodies against HPSE, phospho-RAF, phospho-ERK and phospho-MEK with anti- $\beta$-actin as the control. The blocking steps and dilutions for the assessment of all proteins were performed in 5\% bovine serum albumin. After incubation with horseradish peroxidaseconjugated antibodies (Amersham Pharmacia), the labeled proteins were detected with an ECL-Plus detection system (Amersham Pharmacia). The anti-HPSE antibody was from Abcam, anti phospho-MEK, anti phospho-RAF, phosphoERK and total ERK antibodies were from Cell Signaling. The anti- $\beta$-actin was from Santa Cruz Biotechnology (Santa Cruz, CA, USA).

Cell proliferation assay. CNE-2, CNE-2/shHPSE and $\mathrm{CNE}-2 / \mathrm{NC}$ cells were prepared at a concentration of $1 \times 10^{4}$ cells $/ \mathrm{ml}$ and incubated for 1, 2, 3, 4, 5, 6 and 7 days, respectively. 3-(4,5-Dimethylthiazol-2-yl)-2,5-diphenyltetrazolium bromide (MTT) assay was performed by adding $20 \mu \mathrm{l}$ 
of MTT (Promega) for $4 \mathrm{~h}$. The supernatants were removed and $150 \mu 1$ of DMSO (Sigma) was added to each well. Fifteen minutes later, the absorbance value (OD) of each well was measured with a microplate reader set at $490 \mathrm{~nm}$. All experiments were performed in triplicate.

Plate clone formation assay. Approximately $1 \times 10^{2}$ cells were added to each well (3-cm in diameter) of a 6-well culture plate, and each cell group consisted of three wells. After incubation at $37^{\circ} \mathrm{C}$ for 12 days, the cells were washed twice with PBS and stained with Giemsa solution. The number of colonies containing $\geq 50$ cells was counted under a microscope. Plate clone formation efficiency $=$ (number of colonies/number of cells inoculated) $\mathrm{x} 100 \%$. Three individual experiments were carried out.

Cell motility assay. CNE-1, CNE-2/shHPSE and CNE-2/NC $\left(1 \times 10^{5}\right)$ cells in $0.5 \mathrm{ml}$ serum-free medium were placed in the upper chamber of a transwell, whereas the lower chamber was loaded with $0.8 \mathrm{ml}$ medium containing $10 \%$ FBS. The cells that migrated to the lower chamber were stained with $0.5 \%$ crystal violet, and the total cell number was counted after $24 \mathrm{~h}$ of incubation at $37^{\circ} \mathrm{C}$ with $5 \% \mathrm{CO}_{2}$.

Cell invasion assay. Upper chambers of a 24-well Transwell plate (Corning Incorporated) were coated with 50\% Matrigel (BD Biosciences) in phosphate-buffered saline. CNE-2, CNE-2/shHPSE and CNE-2/NC cells were incubated in the upper chamber. After a 24-h incubation, invaded cells were stained with $0.5 \%$ crystal violet, examined by bright field microscopy, and photographed. The invasion rate was quantified by counting the number of invaded cells in five random fields per chamber under a fluorescence microscope. Data values were summarized from three independent experiments.

Tumorigenicity assay. CNE-2/shHPSE and CNE-2/NC $\left(5 \times 10^{6}\right)$ cells were injected subcutaneously in the flank of 6 to 7-week-old nude mice. Tumor growth was evaluated for 3 weeks after injection and every 7 days thereafter. The tumor volume was determined by measuring the largest (a) and the smallest (b) axis using a caliper, and calculated as $\mathrm{V}=0.5 \mathrm{ab}^{2}$. Mice handling and experimental procedures followed institutional guidelines. All experimental procedures and protocols were approved by our Institutional Animal Care and Use Committee.

Statistical analysis. Unless otherwise stated, the data are presented as means \pm standard error of the mean (SEM). Statistical significance $(\mathrm{P}<0.05)$ was determined by the $\mathrm{t}$-test or analysis of variance (ANOVA) followed by assessment of differences using SPSS 16.0 software (SPSS Inc., Chicago, IL, USA).

\section{Results}

Expression of HPSE in three nasopharyngeal carcinoma cell lines. To investigate the expression of HPSE in nasopharyngeal carcinoma cells, we performed qRT-PCR and western blotting of C666, 6-10B and CNE-2 cells. The mRNA and protein levels of HPSE in the three cell lines are shown in Fig. 1. The
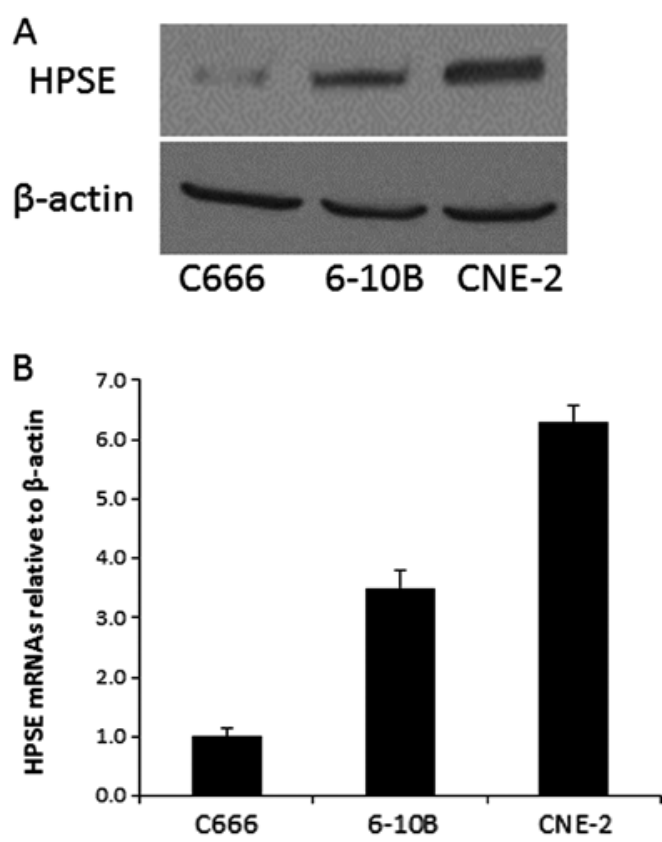

Figure 1. HPSE expression in three human NPC cell lines with different metastatic potential. (A) HPSE protein expression levels in C666, 6-10B and CNE-2 cell lines were determined by western blot analysis. CNE-2 cells exhibited the most markedly increased expression of the HPSE protein as compared to the other cell lines. (B) Quantification of HPSE mRNA expression in the different cells (C666, 6-10B and CNE-2) related to the control as detected by qRT-PCR. CNE-2 cells expressed the highest level of HPSE mRNA. The expression of $\beta$-actin protein and mRNA was also examined and served as controls for sample loading. The image shows a representative result of three independent experiments.

data showed that CNE-2 cells expressed the highest level of HPSE mRNA and protein, and thus, was chosen to further study the biological function of HPSE by RNA interference.

The vector stably expressing HPSE shRNA causes effective and specific downregulation of HPSE expression. The knock down efficiencies of the different HPSE-specific shRNAs in CNE-2 cells were first evaluated using qRT-PCR. Relative HPSE mRNA levels in the individual stable transfectants were normalized against the mRNA level of an internal control gene, $\beta$-actin, performed in the same run. As shown in Fig. $2 \mathrm{~A}$, cells transfected with pGPU6-HPSE-shRNA3 showed a significantly reduced level of HPSE protein when compared with that of the vector control and the negative transfectants, respectively. A reduction in protein was not detectable in cells transfected with pGPU6-HPSE-shRNA1 and pGPU6-HPSEshRNA2. In addition, qRT-PCR analysis (Fig. 2B) showed a marked reduction in the HPSE mRNA levels in the CNE-2 cell line transfecting with HPSE-shRNA3. The above results demonstrated that expression of HPSE was downregulated specifically and effectively by the specific HPSE shRNA, and that different shRNAs showed striking differences in silencing efficiency. Thus, CNE-2 cells transfected with pGPU6-HPSEshRNA3 were named CNE-2/shHPSE, and CNE-2 cells transfected with pGPU6-shNC were named CNE-2/NC.

Effect of HPSE on cell proliferation of CNE-2 cells. The proliferative activity of tumor cells is important in the invasion/metastasis of tumors. After identifying the most effective 
A

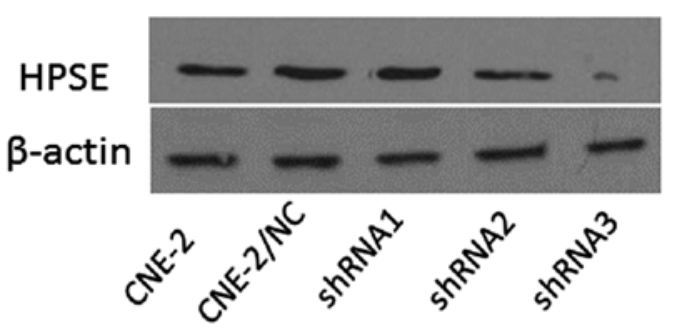

B

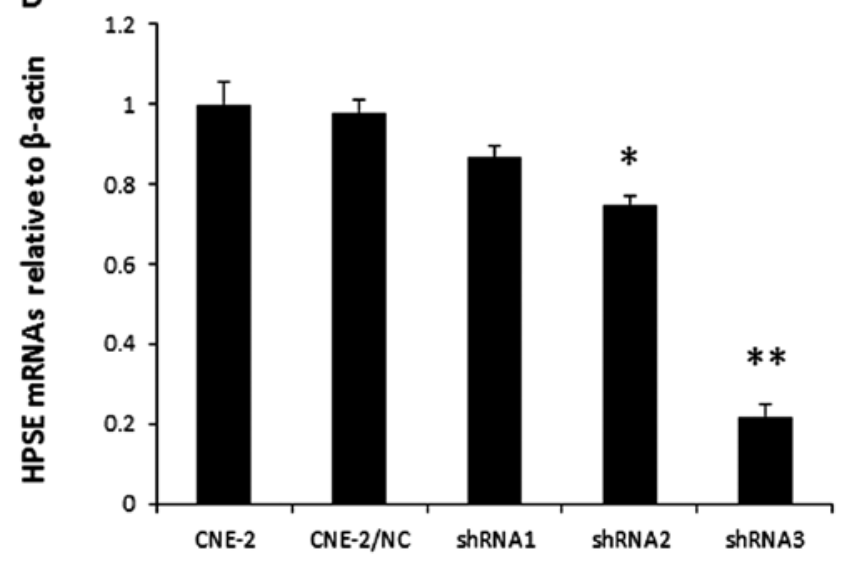

Figure 2. HPSE-specific shRNAs result in the reduction of HPSE mRNA and protein in CNE-2 cells. (A) Levels of HPSE protein were assessed by western blotting. The results revealed that protein production of HPSE in CNE-2 cells was effectively inhibited and most significantly by HPSE-shRNA3 interference. (B) HPSE mRNA expression in CNE-2 cells stably transfected with HPSE-shRNA3 was decreased most significantly when compared to the CNE-2/NC and CNE-2 cells ( $\left.{ }^{*} \mathrm{P}<0.05,{ }^{* *} \mathrm{P}<0.01\right)$. The expression of $\beta$-actin mRNA and protein was also examined and served as controls for sample loading.

HPSE-specific shRNA transfectant (CNE-2/shHPSE), we immediately examined cell proliferative activity of the transfected cells using MTT assay. As known in Fig. 3A, the growth of CNE-2 cells in vitro was markedly inhibited after the transfection of pGPU6-HPSE-shRNA3 $(\mathrm{P}<0.05)$. This indicates a positive relation between the expression of HPSE and the rate of NPC cell growth.

Effect of the silencing of HPSE using shRNA on the colony formation potential of CNE-2 cells. In order to document the ability of CNE-2/shHPSE cells to form colonies, single-cell suspensions were plated at a density of 100 cells in $30-\mathrm{mm}$ culture dishes. As shown in Fig. 3B, after 12 days, CNE-2/ shHPSE cells, compared with CNE2 and MHCC97-H/negative-shRNA cells, exhibited a significant reduction in their ability to form colonies, and their ability to form colonies was correlated with HPSE expression $(\mathrm{P}<0.05)$ (Fig. 3C).

Effect of HPSE on the migration and invasion of CNE-2 cells. CNE-2/shHPSE cells displayed a marked decrease in the migratory ability as comparing with either CNE-2/NC or $\mathrm{CNE} / 2$ cells $(\mathrm{P}<0.01$, respectively). As shown in Fig. $4 \mathrm{~A}$ and $\mathrm{B}$, the migration rate of the CNE-2/shHPSE cells was $25.9 \%$, while the rates of the CNE-2 and CNE-2-NC groups were 100 and $97 \%$, respectively. Inhibition of HPSE caused significantly attenuated migration of CNE-2 cells.
A

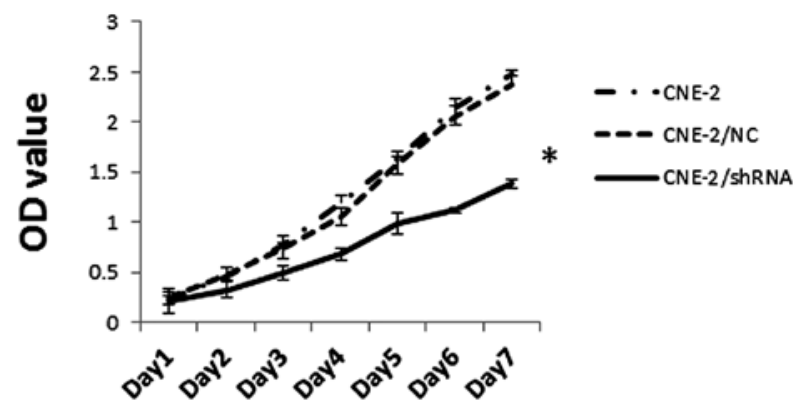

B

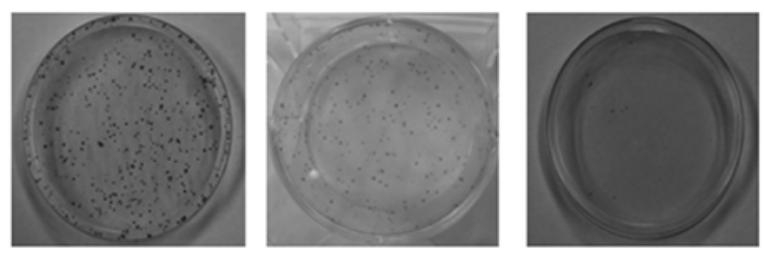

$\mathrm{C}$

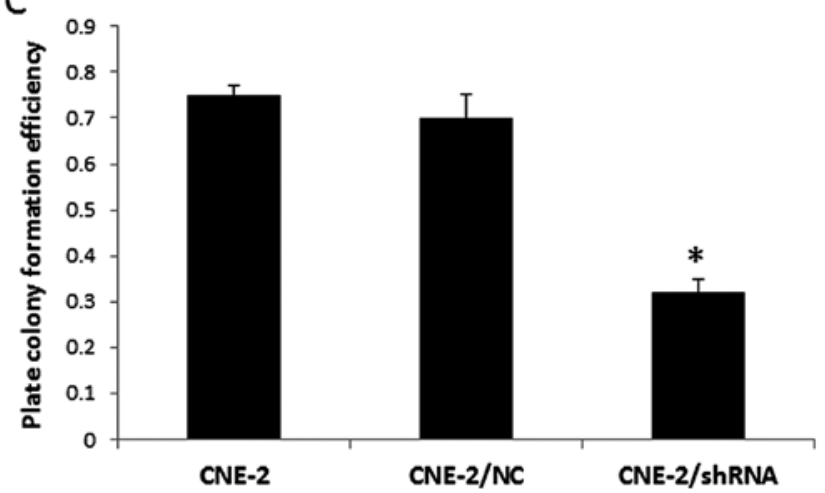

Figure 3. HPSE gene silencing suppresses cell proliferation and colony formation potential in vitro. (A) Proliferation of CNE-2 cells stably transfected with HPSE/shRNA1, negative-shRNA and vector were analyzed by an MTT assay as previously described. The number of viable cell was assessed using an MTT assay at 1, 2, 3, 4, 5, 6 and 7 days, respectively. Each sample was tested in triplicates, and the results were reported as OD readings (means \pm SD). CNE-2/shHPSE cells showed marked inhibition of cell proliferation when compared with both the CNE-2/NC and CNE-2/vector cells ( $\mathrm{P}<0.05)$. (B) After 12 days of culture, the cells were stained with MayGiemsa and we counted those $<1 \mathrm{~mm}$. Values represent the means \pm SD of at least three independent experiments. (C) Statistical analysis showed that the efficiency of CNE-2/shHPSE cells to form colonies was significantly lower than that of the CNE-2 and CNE-2/NC cells ( $\mathrm{P}<0.05)$.

Invasion assays were carried out using Matrigel-coated Transwell culture chambers. CNE-2/shHPSE cells exhibited much lower invasive abilities than either the CNE-2 or CNE-2/NC cells ( $\mathrm{P}<0.01$, respectively) (Fig. 4C and D). The invasion rates of the CNE-2, CNE-2/NC and CNE-2/shHPSE cells were 100,98 and $34.6 \%$, respectively. Suppression of HSPE led to significantly reduced invasive ability of the CNE-2 cells.

Effects of HSPE inhibition on the expression of MARK transduction. Western blot analysis was applied to detect the expression of components of MARK transduction in CNE/2, CNE-2/NC and CNE-2/shHPSE cells. Inhibition of HPSE 
A
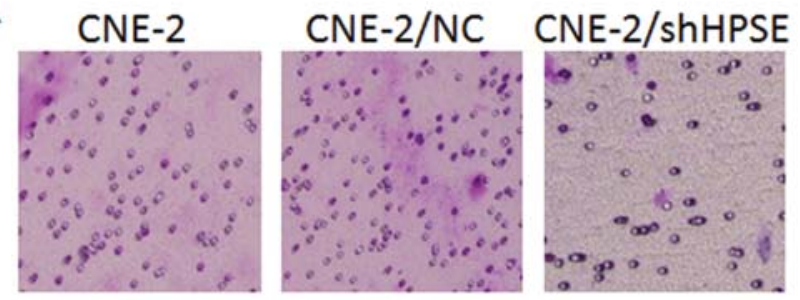

B

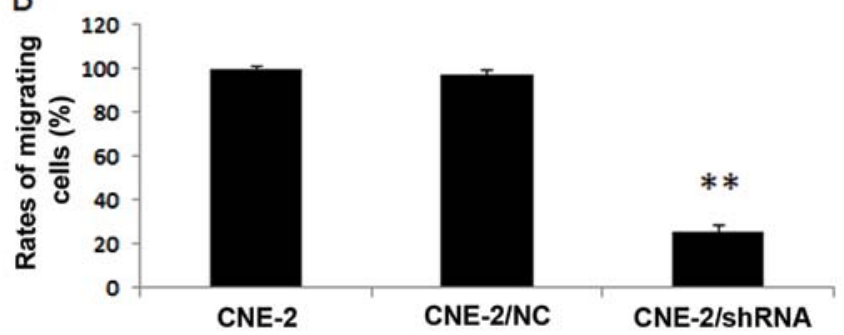

C
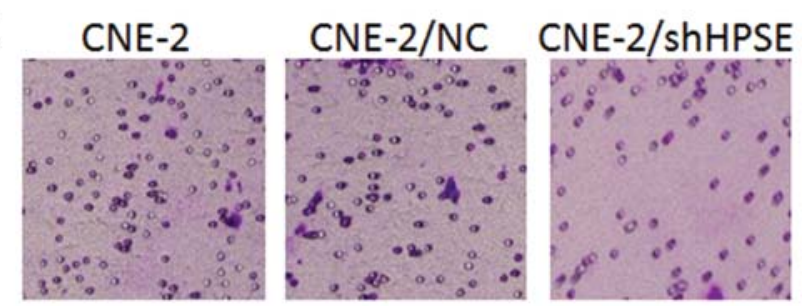

\section{D}

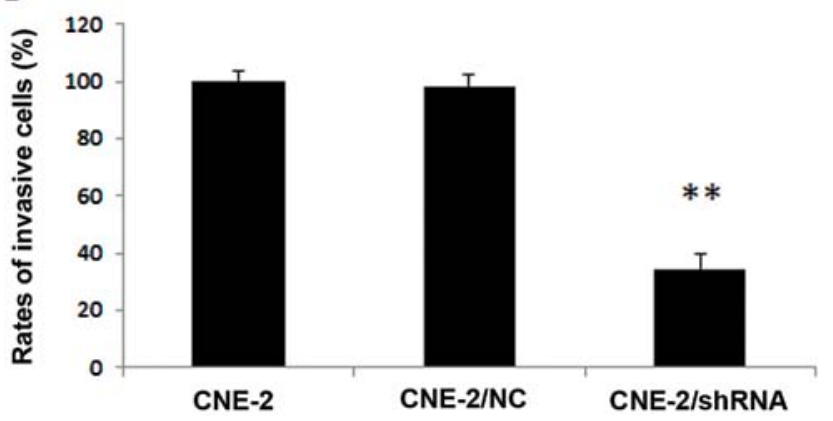

Figure 4. Effect of HPSE knockdown on the invasive and migratory potential of NPC cells. (A) In vitro migration assay was carried out to compare and quantify the migratory capacity of CNE-2, CNE-2/NC and CNE-2/shHPSE cells. Results are representative of three independent experiments and bars represent the means \pm SD. (B) Quantitative Transwell migration assay. The $y$-axis represents the number of migrating cells. Data are the average numbers of cells that migrated in a representative experiment, measured in triplicate and presented as the means \pm SD (magnification, $\mathrm{x} 200)\left({ }^{* * *} \mathrm{P}<0.01\right.$ vs. CNE2). (C) HPSE-silenced cells showed decreased invasive ability that was correlated with HPSE expression. (D) Quantitative Transwell invasion assay. The $y$-axis represents the number of invasive cells. Data are the average numbers of cells that migrated in a representative experiment, measured in triplicate and presented as means $\pm \mathrm{SD}$ (magnification, $\mathrm{x} 200)\left({ }^{* *} \mathrm{P}<0.01\right.$ as compared to CNE2).

resulted in a significant decrease in $\mathrm{p}-\mathrm{RAF}, \mathrm{p}-\mathrm{RAF}$ and $\mathrm{p}-\mathrm{MEK}$ expression. Protein levels of p-RAF, p-ERK and p-MEK in the CNE-2/shHPSE cells were lower than these levels in the control groups (CNE-2 and CNE-2/NC) (Fig. 5).

HPSE gene silencing suppresses cell proliferation in vivo. The effect of HPSE on in vivo tumor growth was assessed by the subcutaneous injection of CNE-2/shHPSE and CNE-2/NC cells in nude mice for 21 days. As shown in Fig. 6, a marked

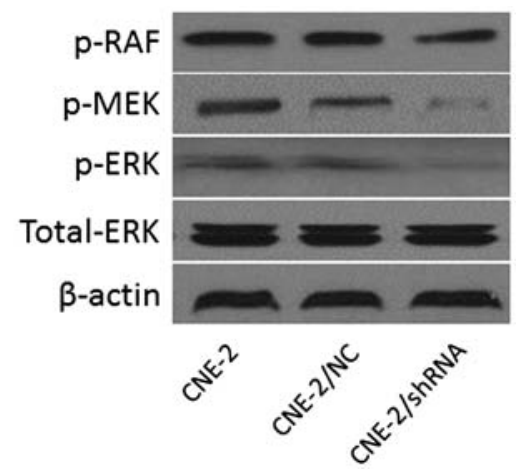

Figure 5. Downregulation of HPSE inhibited the Raf/MEK/ERK pathway. Total cellular proteins were collected and subjected to western blot analysis. Stable downregulation of HPSE reduced the protein level of p-RAF, p-MEK and p-ERK.
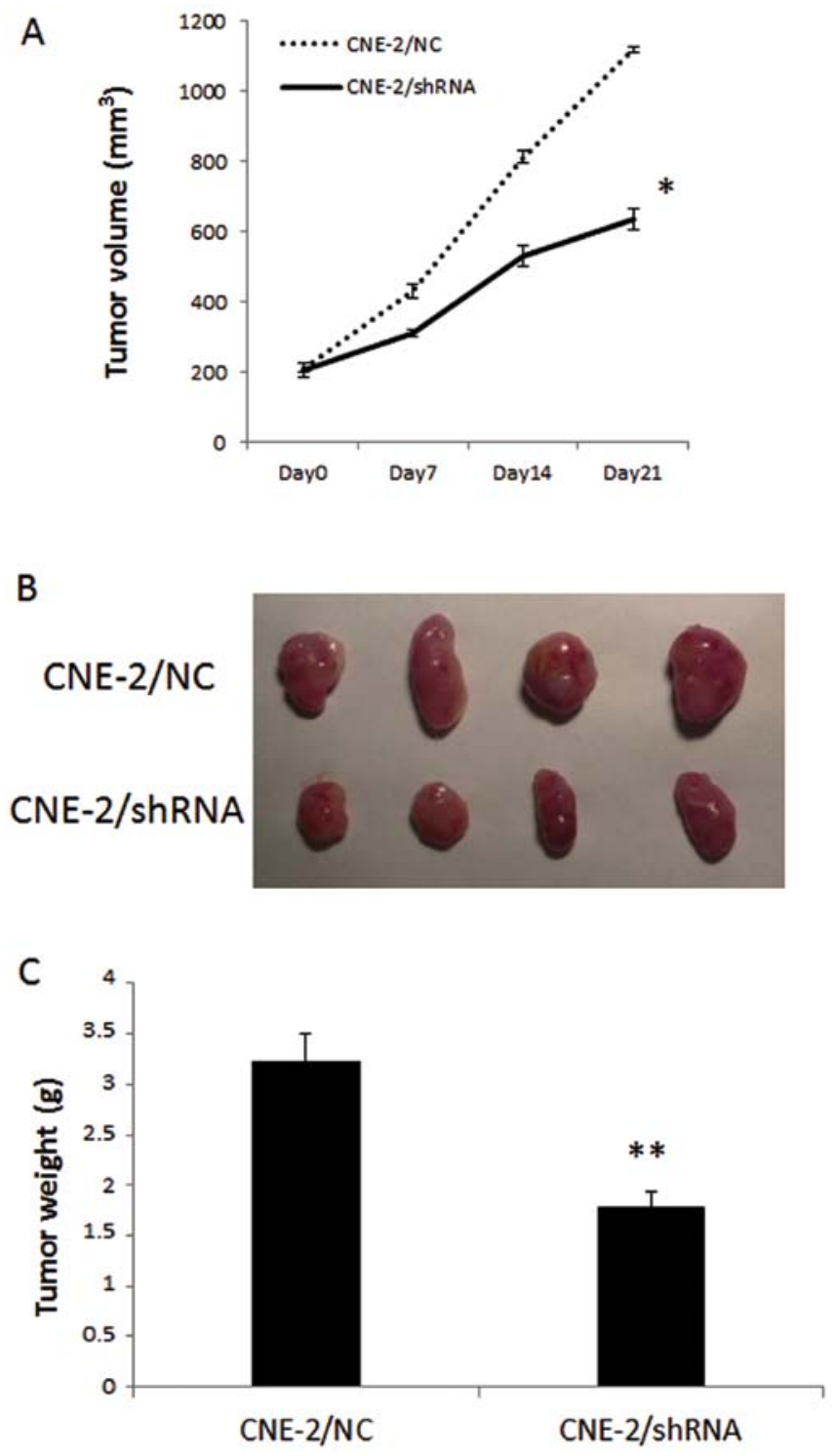

Figure 6. Knockdown of HPSE in the CNE-2 cells retards tumorigenicity in nude mice. CNE-2/shHPSE and the control (CNE-2/NC) cells were injected subcutaneously into the right flanks of nude mice. (A) CNE-2/shHPSE cell tumors showed a significant decrease in growth rate, as compared to CNE-2/NC cell tumors $\left({ }^{*} \mathrm{P}<0.05\right)$. (B) On the 21 st day after injection the tumors were dissected and weighed $(n=4)$. (C) Average tumor weights $(\mathrm{n}=4)$ of the CNE-2/shHPSE and CNE-2/NC groups were $1.78 \pm 0.16$ and $3.23 \pm 0.27$ g, respectively $\left({ }^{* *} \mathrm{P}<0.01\right)$. 
reduction in tumor size in the CNE-2/shHPSE groups was observed as compared with that of the control group $(\mathrm{P}<0.05)$. By day 21 after cell injection, the average tumor weight $(n=4)$ of the CNE-2/shHPSE and CNE-2/NC groups was $1.78 \pm 0.16$ and $3.23 \pm 0.27 \mathrm{~g}$, respectively $(\mathrm{P}<0.01)$, indicating that knockdown of HPSE in NPC cells reduced their tumorigenic potential.

\section{Discussion}

In the present study, we showed that overexpression of HPSE has an important role in the progression of NPC. These findings suggest that HPSE is a major contributor to the proliferation, invasion and migration of NPC cells.

HPSE enzymatic activity, capable of cleaving glucuronidic linkages and releasing polysaccharide chains resistant to further degradation by the enzyme, was first identified by Ogren and Lindahl (9). The activity of HPSE was shown to be related with the metastatic potential of tumor cells such as B16 melanoma and T-lymphoma $(10,11)$. These early observations gained substantial support from many subsequent studies that clearly indicate that HPSE not only enhances cell dissemination, but also promotes the establishment of a vascular network which accelerates primary tumor growth and provides a gateway for invading metastatic cells $(12,13)$. In addition, the clinical significance of the enzyme in tumor development emerged from a systematic evaluation of HPSE expression in primary human tumors, including those of the bladder (14), colon (15), stomach (16), breast (17), oral cavity (18), esophagus (19), pancreas (20) and brain (21), and in multiple myeloma and acute myeloid leukemia $(22,23)$. It has also been reported that HPSE is upregulated in essentially all human tumors examined. Notably, increased HPSE levels are often associated with reduced patient survival, increased carcinoma metastasis and high microvessel density $(12,13)$.

Since HPSE plays an indispensable role in the progression of cancer, numerous studies have focused on the development of HPSE inhibitors, including neutralizing antibodies, peptides, small molecules, modified non-anticoagulant species of heparin, as well as several other polyanionic molecules, such as laminaran sulfate, suramin and PI-88. These inhibitors that reduce HPSE expression in cancer cells could significantly decrease their metastatic properties, signifying the importance of HPSE in cancer cell dissemination (24-27). However, because of the diverse biologic activities of these compounds, the mechanism of their antitumor activity and its relation to HPSE inhibition are not straightforward. Moreover, these compounds may produce several non-specific and undesirable effects when they interact with the molecules which are distributed on the cell surface (15-17). Thus, novel approaches are needed to inhibit the effect of HPSE in cancer progression.

RNAi is a specific, potent, and highly successful approach for loss-of-function studies in virtually all eukaryotic organisms (28). It has been identified in several reports that HPSE shRNA significantly downregulates both HPSE protein and mRNA expression, and inhibits proliferation and invasion of tranfected cells in different tumor models, such as breast cancer (29), hepatocellular carcinoma (30) and gastric tumors (31). In the present study, CNE-2 was chosen as the RNA interfering subject, as it demonstrated increased levels of
HPSE as compared with other NPC cell lines. The cytological results indicated a relationship between attenuated HPSE expression and cell proliferation, and together with further in vivo experiments, support our hypothesis that HPSE may be a direct factor contributing to the carcinogenesis and development of NPC by regulating cellular proliferation. In addition, low expression of HPSE is also associated with a marked decrease in invasive capability of CNE-2 cells in vitro. The above results suggest a possible reason for the poor outcomes observed in NPC patients overexpressing HPSE.

The MAPK signaling pathway is a major determinant in the control of diverse cellular processes such as proliferation, survival, differentiation and motility and is associated with tumor development (32). Our results indicate that the antiproliferative effects of HPSE on NPC cells are partially mediated by MAPK signaling. Suppression of HPSE in NPC cells overexpressing HPSE inhibited cell proliferation associated with a decrease in phosphorylation of RAF, MEK and ERK. However, we noted that even after knockdown of HPSE, the NPC cells still possessed the capabilities of invasion and metastasis. We believe that other factors, such as uPA and MMPs, also influence these characteristics of NPC, which warrants further investigation.

In summary, we demonstrated that suppression of HPSE results in MAPK signaling pathway inactivation in NPC cells, consequently inhibiting cell proliferation and invasion. Together with previous reports that interference of HPSE expression regulates at least two major signaling pathways (Wnt/ $\beta$-catenin and TGF- $\beta$ ) in cancer, we suggest that interference of HPSE expression and function exert broad-spectrum biologic effects that may be beneficial for the treatment of NPC. Further studies of the mechanisms by which HPSE regulates multiple signaling pathways may reveal other points of intervention for this important target.

\section{Acknowledgements}

We gratefully thank Chengwei Lv from the Cancer Biotherapy Center for the collection and maintenance of the CNE-2, 6-10B and C666 cell lines used in this research. The project was supported by the National Natural Science Foundation of China (grant no. 81001212), the Science and Technology Foundation of Zhejiang Province (grant no. 2012C33011), Foundation of State Key Laboratory of Oncology in South China (grant no. HN2011-03), Zhejiang Provincial Chinese Medicine Research Foundation (grant no. 2011ZQ012), and the Natural Science Foundation of Guangdong (grant no. s2012010008209).

\section{References}

1. Jemal A, Bray F, Center MM, Ferlay J, Ward E and Forman D: Global cancer statistics. CA Cancer J Clin 61: 69-90, 2011.

2. Wei WI and Sham JS: Nasopharyngeal carcinoma. Lancet 365: 2041-2054, 2005

3. Sanderson RD, Yang Y, Suva LJ and Kelly T: Heparan sulfate proteoglycans and heparanase - partners in osteolytic tumor growth and metastasis. Matrix Biol 23: 341-352, 2004

4. Simizu S, Ishida K and Osada H: Heparanase as a molecular target of cancer chemotherapy. Cancer Sci 95: 553-558, 2004

5. Edovitsky E, Elkin M, Zcharia E, Peretz T and Vlodavsky I: Heparanase gene silencing, tumor invasiveness, angiogenesis, and metastasis. J Natl Cancer Inst 96: 1219-1230, 2004. 
6. Zheng L, Jiang G, Mei H, et al: Small RNA interferencemediated gene silencing of heparanase abolishes the invasion, metastasis and angiogenesis of gastric cancer cells. BMC Cancer 10: 33, 2010.

7. Lerner I, Baraz L, Pikarsky E, et al: Function of heparanase in prostate tumorigenesis: potential for therapy. Clin Cancer Res 14: 668-676, 2008

8. Bar-Sela G, Kaplan-Cohen V, Ilan N, Vlodavsky I and Ben-Izhak O: Heparanase expression in nasopharyngeal carcinoma inversely correlates with patient survival. Histopathology 49: 188-193, 2006.

9. Ogren S and Lindahl U: Cleavage of macromolecular heparin by an enzyme from mouse mastocytoma. J Biol Chem 250: 2690-2697, 1975.

10. Nakajima M, Irimura T, Di Ferrante D, Di Ferrante $\mathrm{N}$ and Nicolson GL: Heparan sulfate degradation: relation to tumor invasive and metastatic properties of mouse B16 melanoma sublines. Science 220: 611-613, 1983.

11. Vlodavsky I, Fuks Z, Bar-Ner M, Ariav Y and Schirrmacher V: Lymphoma cell-mediated degradation of sulfated proteoglycans in the subendothelial extracellular matrix: relationship to tumor cell metastasis. Cancer Res 43: 2704-2711, 1983.

12. Ilan N, Elkin M and Vlodavsky I: Regulation, function and clinical significance of heparanase in cancer metastasis and angiogenesis. Int J Biochem Cell Biol 38: 2018-2039, 2006.

13. Vreys V and David G: Mammalian heparanase: what is the message? J Cell Mol Med 11: 427-452, 2007.

14. Zhao W, Wang XS, Niu HT, Wang LL, Han BM and Xia SJ: Clinical relevance of heparanase mRNA expression in bladder cancer and its usefulness as a detection marker in voided urine. Mol Med Rep 2: 327-331, 2009.

15. Nobuhisa T, Naomoto Y, Ohkawa T, et al: Heparanase expression correlates with malignant potential in human colon cancer. J Cancer Res Clin Oncol 131: 229-237, 2005.

16. Chen JQ, Zhan WH, He YL, et al: Relationship between heparanase mRNA expression in human gastric cancer and its clinicopathological features. Zhonghua Zhong Liu Za Zhi 26 609-611, 2004 (In Chinese).

17. Theodoro TR, de Matos LL, Sant AA, et al: Heparanase expression in circulating lymphocytes of breast cancer patients depends on the presence of the primary tumor and/or systemic metastasis. Neoplasia 9: 504-510, 2007.

18. Leiser Y, Abu-El-Naaj I, Sabo E, et al: Prognostic value of heparanase expression and cellular localization in oral cancer. Head Neck 33: 871-877, 2011.

19. Brun R, Naroditsky I, Waterman M, et al: Heparanase expression by Barrett's epithelium and during esophageal carcinoma progression. Mod Pathol 22: 1548-1554, 2009.
20. Quiros RM, Rao G, Plate J, et al: Elevated serum heparanase-1 levels in patients with pancreatic carcinoma are associated with poor survival. Cancer 106: 532-540, 2006.

21. Hong X, Nelson KK, DeCarvalho AC and Kalkanis SN Heparanase expression of glioma in human and animal models. J Neurosurg 113: 261-269, 2010.

22. Kelly T, Miao HQ, Yang Y, et al: High heparanase activity in multiple myeloma is associated with elevated microvessel density. Cancer Res 63: 8749-8756, 2003.

23. Bitan M, Polliack A, Zecchina G, et al: Heparanase expression in human leukemias is restricted to acute myeloid leukemias. Exp Hematol 30: 34-41, 2002.

24. Nakajima M, DeChavigny A, Johnson CE, Hamada J, Stein CA and Nicolson GL: Suramin. A potent inhibitor of melanoma heparanase and invasion. J Biol Chem 266: 9661-9666, 1991.

25. Miao HQ, Liu H, Navarro E, Kussie P and Zhu Z: Development of heparanase inhibitors for anti-cancer therapy. Curr Med Chem 13: 2101-2111, 2006.

26. Miao HQ, Elkin M, Aingorn E, Ishai-Michaeli R, Stein CA and Vlodavsky I: Inhibition of heparanase activity and tumor metastasis by laminarin sulfate and synthetic phosphorothioate oligodeoxynucleotides. Int J Cancer 83: 424-431, 1999.

27. Karoli T, Liu L, Fairweather JK, et al: Synthesis, biological activity, and preliminary pharmacokinetic evaluation of analogues of a phosphosulfomannan angiogenesis inhibitor (PI-88). J Med Chem 48: 8229-8236, 2005.

28. Fjose A, Ellingsen S, Wargelius A and Seo HC: RNA interference: mechanisms and applications. Biotechnol Annu Rev 7: $31-57,2001$

29. Zhang ZH, Chen Y, Zhao HJ, Xie CY, Ding J and Hou YT: Silencing of heparanase by siRNA inhibits tumor metastasis and angiogenesis of human breast cancer in vitro and in vivo. Cancer Biol Ther 6: 587-595, 2007.

30. Zhang Y, Li L, Wang Y, et al: Downregulating the expression of heparanase inhibits the invasion, angiogenesis and metastasis of human hepatocellular carcinoma. Biochem Biophys Res Commun 358: 124-129, 2007.

31. Jiang G, Zheng L, Pu J, et al: Small RNAs targeting transcription start site induce heparanase silencing through interference with transcription initiation in human cancer cells. PLoS One 7: e31379, 2012

32. De Luca A, Maiello MR, D'Alessio A, Pergameno $M$ and Normanno N: The RAS/RAF/MEK/ERK and the PI3K/AKT signalling pathways: role in cancer pathogenesis and implications for therapeutic approaches. Expert Opin Ther Targets 16 (Suppl 2): S17-S27, 2012. 